\title{
MR Enterography in Young Children: Triks and Tips
}

\author{
Emanuele Casciani*1, Giovanni Di Nardo ${ }^{1,2}$ \\ ${ }^{1}$ Salvator Mundi International Hospital, UPMC, Italy \\ ${ }^{2}$ Pediatric Gastroenterology Unit, AORN Santobono-Pausilipon, Italy
}

Received: January 31, 2018; Published: February 16, 2018

*Corresponding author: Emanuele Casciani, Salvator Mundi International Hospital, Viale delle Mura Gianicolensi 67, 00152 Rome, Italy, Tel: 390658896555;

Email: emanuelecasciani@gmail.com

\begin{abstract}
Magnetic resonance enterography (MRE) is an increasingly important pediatric imaging modality that is most often used to evaluate mostly inflammatory bowel disease (IBD), while sparing children from potential risks of ionizing radiation exposure. The younger children have to be investigating under anesthesia. To avoid anesthesia, patient preparation prior to imaging and protocol optimization are essential to consistently obtaining high-quality MR images of the young children bowel.
\end{abstract}

Keywords: Magnetic Resonance Enterography; Pediatric

\section{Introduction}

Magnetic resonance enterography (MRE) is an increasingly important pediatric imaging modality and is currently a firstline imaging tool for assessing the extent of inflammatory bowel disease (IBD), providing information both inside and outside the bowel lumen [1]. MRE has also proven useful for evaluating a variety of other diseases beyond IBD, such as known or suspected bowel masses or polyps, bowel obstruction (nonacute setting), and obscure gastrointestinal bleeding [2]. However, challenges exist when imaging young children, including enter into a tube, inability to tolerate and hold still for long examinations, insufficient intake of oral contrast material, lack of cooperation with breath hold instructions, and trauma during and change in behavior after placement of intravenous (IV) lines for gadolinium administration. All these challenges can generate poor tolerance and movement artifacts during the MR examinations. Patient preparation prior to imaging and protocol optimization are essential to consistently obtaining high-quality MR images of the young children bowel, especially in children ages 4 to 8 years. In these young patients it is possible to avoid anesthesia or Computed Tomography by using an optimized MR protocol.

\section{Patient Preparation}

\section{First Step: Reduced Anxiety}

Informing the child and parent/caregiver about what MRE entails is very helpful in alleviating patient anxiety and improving tolerance and compliance, resulting in a high-quality diagnostic
MRE. Some centers obtained optimal results using mock MRI or simulator that permitted to prepare the children, by familiarizing them with the environment, sounds, and equipment, reducing the number of general anesthesia in younger patients [3]. Moreover, IV cannulation is a major cause of anxiety in young patients presenting for MRI examination. In our experience, it is helpful to separate the procedures of IV placement and the MR exam by placing a peripheral intravenous catheter before to arrive in the radiology department.

In addition to routine MR imaging safety screening, the patient's ability to hold still (as our routine MRE examination lasts about 3040 minutes) and perform adequate breath holds on command are evaluated before the MR examination. Breath holds may be timed to get an idea how long an individual can hold his or her breath to guide the MR imaging technologist in pulse sequence optimization. If it is determined that a child likely will not be able to successfully complete the examination awake, the examination is rescheduled into the future to be performed under general anesthesia.

\section{Second Step: Adequate Bowel Distention}

No special diet is required prior to MRE and patients should abstain from all food and drinks for $4-6 \mathrm{~h}$ prior to the study. Small bowel distension is crucial to obtain high-quality MRE exams. Compliance can be optimized by giving the patient input into choice of flavoring, keeping drinks chilled and giving lots of positive encouragement. Different protocols and timing have been proposed 
for the ingestion of the hyperosmolar solutions [4]. In our institute, patients started to drink a polyethylene glycol (PEG)-water solution with a taste of lemon or orange (dose: $10 \mathrm{~mL} / \mathrm{kg}$ body weight) 90 minutes before the examination. If the child was unable to drink the entire dose of oral contrast, we asked them to drink water or juice (or a mixture of both) according to the child's preference in order to reach the target volume of enteric fluid. Good distension of the bowel is aided by drinking boluses quickly rather than as slow continuous sips. The child was asked to lie in the right lateral position $10 \mathrm{~min}$ before the exam, to facilitate gastric emptying.

\section{Third Step: Remain into the Tube}

Before starting the exam, it is important to ask the child to empty their bladder to reduce compression of bowel loops from an over-distended bladder, and this also reduces patient discomfort. It is also helpful to reassure children that passage of loose stool at this time is normal. Supine position is better tolerated in pediatric patients, unlike adults. One parent can remain in the imaging room with the patient if so desired after full safety screening of the parent involved. Recently MR-compatible audiovisual distraction devices have been utilized to improve procedure tolerance of the pediatric patients. This technology uses fiber optic cables that do not interfere with the MR scan and allow patients to watch their favorite movies during their MRI. Courtier J, et al. [5] showed that MRE can be successfully performed in children ages 4 to 7 years using an abbreviated protocol in combination with MR video goggles. This approach can reduce time required for the diagnostic scan, the number of patients who have require anesthesia, and costs.

\section{Protocol optimization}

Diagnostic quality pediatric MRE can be performed on both 1.5 T and 3-T MR scanners. Regardless to the strength field MR, it is very important to tailor the MR protocol to the features of the patient, including patient's size, and breath holds ability. Multiple pediatric MRE protocols have been described in the literature, most of which rely on a variety of axial and coronal pre-contrast and post-contrast pulse sequences acquired after the administration of oral contrast material [1-6]. Antiperistaltic medications, such as intravenous glucagon or hyoscine-N-butylbromide, can be used to reduce bowel peristalsis and improve image quality. Gastrointestinal effect begin very fast $(<1$ minute) and the duration is $6.8+/-5.3$ minutes for hyoscine-N-butylbromide and $18.3+/-7$ minutes for glucagon. For this reason it is recommended use two or three doses each of 0.3 $\mathrm{mg} / \mathrm{kg}$ to a maximum of $20 \mathrm{mg}$ per dose.

To maximize image quality (signal-to-noise ratio and spatial resolution) while minimizing examination length (using parallel imaging), multichannel surface coils are typically utilized. Usually a comprehensive MR examination of the small bowel usually include: 1) high-resolution ultra-fast sequences such as balanced steady- state free precession (SSFP) and Half-Fourier single-shot turbo spin echo (HASTE) sequences with and without fat suppression; 2) fat-suppressed three-dimensional (3D) T1-weighted breath-hold gradient-echo images of the abdomen and pelvis before and after intravenous gadolinium-based contrast material administration; 3) Diffusion-weighted imaging (DWI). Recent studies have shown that DWI may be useful for detecting active bowel inflammation [7-8]. Despite these advances, DWI has inherent disadvantages, including low spatial resolution and difficulty with exact anatomic localization of suspected areas of abnormality. This problem can be eliminate using colorized, fused DWI and anatomic Single-Shot Fast Spin Echo (SSFSE) T2 images to capitalize on the additional functional information contained in DWI images without losing anatomic detail present in high-resolution T2 weighted images [9].

\section{Conclusion}

Younger patients could be very difficult to scan in MR. Patient preparation prior to imaging and protocol optimization are essential to consistently obtaining high-quality MR images of the young children bowel, especially in children ages 4 to 8 years. This is very important to avoid anesthesia in MR or to use radiation technique, as Computed Tomography.

\section{References}

1. Yoon HM, Suh CH, Kim JR, Lee JS, Jung AY, et al. (2017) Diagnostic Performance of Magnetic Resonance Enterography for Detection of Active Inflammation in Children and Adolescents With Inflammatory Bowel Disease A Systematic Review and Diagnostic Meta-analysis. JAMA Pediatr 171(12): 1208-1216.

2. Casciani E, Di Nardo C, Chin S, Masselli G, Polettini E, et al. (2017) MR Enterography in paediatric patients with obscure gastrointestinal bleeding. European Journal of Radiology 93: 209-216.

3. Carter AJ, Greer ML, Gray SE, Ware RS (2010) Mock MRI: reducing the need for anaesthesia in children. Pediatric Radiology 40(8): 1368-1374.

4. M-LC Greer (2016) How we do it: MR enterography. Pediatr Radiol 46(6): 818-828.

5. Courtier J, Cardens A, Tan C, Towne M, Rhee SJ, et al. (2015) Nonanesthesia Magnetic Resonance Enterography in Young Children: Feasibility, Technique, and Performance. JPGN 60(6): 754-761.

6. Mollard BJ, Smith EA, Dillman JR (2015) Pediatric MR enterography: technique and approach to interpretation-how we do it. Radiology 274(1): 29-43.

7. AlSabban Z, Church P, Moineddin R, et al. (2016) Accuracy and interobserver agreement of diffusion-weighted imaging in pediatric inflammatory bowel disease. Clinical Imaging 41(1): 14-22.

8. Dilmann JR, Smith EA, Sanchez R, Adler J, Fazeli S, et al. (2016) DWI in Pediatric Small-Bowel Crohn Disease: Are Apparent Diffusion Coefficients Surrogates for Disease Activity in Patients Receiving Infliximab Therapy? AJR Am J Roentgenol 207(5): 1002-1008.

9. Ehman EC, Phelps AS, Ohliger MA, Rhee SJ, MacKenzie JD, et al. (2016) Detection of bowel inflammation with fused DWI/T2 images versus contrast-enhanced images in pediatric MR enterography with histopathologic correlation. Clin Imaging 40(6): 1135-1139. 


\section{(C) (i) This work is licensed under Creative} Submission Link: http://biomedres.us/submit-manuscript.php

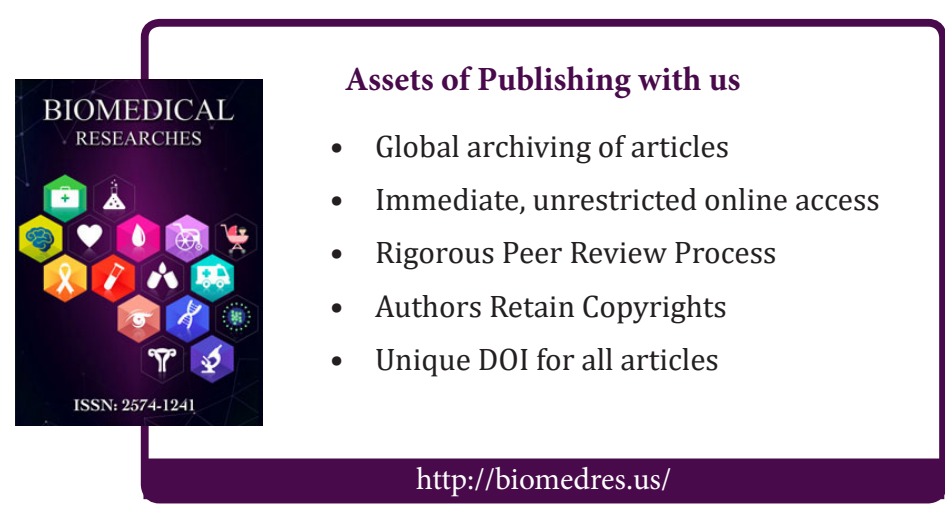

